\title{
User-Based Evaluation of a Data-Driven Medical Education Platform
}

\author{
Dimitrios ZIKOS ${ }^{\mathrm{a}, 1}$ and Cassie E. SPRAGUE ${ }^{\mathrm{a}}$ \\ ${ }^{\text {a } C e n t r a l ~ M i c h i g a n ~ U n i v e r s i t y ~}$
}

\begin{abstract}
TrainCoMorb is an online data-driven training platform for medical students and residents who can practice recognizing comorbidities and their attributable risk for negative hospital outcomes. This is a subsequent cross-sectional evaluation study designed to examine four dimensions of the platform (navigation, usefulness, validity, features) and their association with external factors (age, experience in simulation systems, opinion about data-driven education). Eighteen medical residents participated in a scenario-based evaluation session and completed an online survey. The residents evaluated the four composite dimensions with scores between 3.77 and 4.15 (5-scale) and thought highly of data-driven medical education. Those more familiar with clinical simulation systems, and more positive about data-driven education, evaluated the "usefulness", "validity", and "features" dimensions with higher scores. TrainComorb is intended to be a supplementary tool for the education of future physicians, and this user-based evaluation study provided positive feedback that it could serve its intended scope.
\end{abstract}

Keywords. evaluation, health informatics, medical education, data-driven

\section{Introduction}

TrainCoMorb (Training with Comorbidities) is an online data-driven training platform for medical students and residents who can practice recognizing comorbidities and their attributable risk for negative outcomes. A comorbidity is the presence of one or more diseases co-occurring with a principal diagnosis $\left(\mathrm{D}_{\mathrm{x}}\right)$ [1]. The authors of this study led the development of TrainCoMorb, which was completed in the year 2020 as a joint work between the College of Health Sciences and College of Medicine, at the Central Michigan University (CMU). With the use of TrainCoMorb, trainees (medical students and residents) can create scenarios of comorbidities in a computer assisted manner, to have at their disposal dynamically updated realistic snapshots of patient safety outcomes (hospital mortality, hospital acquired conditions) and the length of stay. The backend of the system is a relational database with 11 million inpatient records. The link to the app is: https://floating-caverns-75251.herokuapp.com. The system design and its clinical significance are explained in [2].

This present paper describes a subsequent study that was designed to evaluate the TrainComorb platform by the intended target audience (medical residents) and to examine several external factors associated with their perceptions about the platform.

${ }^{1}$ Corresponding Author, Dimitrios Zikos, Central Michigan University, Rowe Hall 208c, Mt. Pleasant MI 48858, United States; E-mail: zikos1d@cmich.edu. 


\section{Methods}

\subsection{Survey design and deployment}

The survey included 17 questions which explored the following dimensions: (i) System Navigation, (ii) Usefulness, (iii) Validity of information, (iv) System features. These are typical evaluation components that are examined in health informatics research [3] and in software evaluation [4]. The residents were also asked to provide their opinion about data-driven medical education. The questions used a 5-category Likert scale (Strongly Agree...Strongly Disagree). The survey also asked about the experience with use of computers, exposure with medical simulation systems, and their demographics.

After the study design protocol received approval by the Institutional Review Board, the director of Internal Medicine, Central Michigan University, made a formal invitation and eighteen internal medicine residents agreed to participate. Each resident was compensated with the amount $\$ 100$ upon participation. The principal investigator of the study was not disclosed to the participants to avoid authority bias. After a 15-minute introduction of the platform, the residents were asked to complete four scenarios where they would create, step-by-step, patient comorbidity profiles (Table 1). Upon completion of the four scenarios, the participants were asked to follow a SurveyMonkey link and complete the evaluation survey.

\subsection{Internal Validity and Statistical Analysis}

To examine the internal validity of the four dimensions, Cronbach's Alpha statistic was used (Table 2). The separate questions that were designed to examine each dimension, were found to have an alpha score between 0.704 (navigation dimension) and 0.908 (features dimension). Finally, four questions were used to calculate the composite dimension 'Opinion about data driven medical education' (alpha $=0.84)$. The composite score of each new dimension was calculated as the mean value of the individual items. Since the sample size was relatively small, data analysis was conducted at the $90 \%$ of statistical significance. The analysis was completed with SPSS version 26 [5].

Table 1. Scenarios that the residents completed during the evaluation session

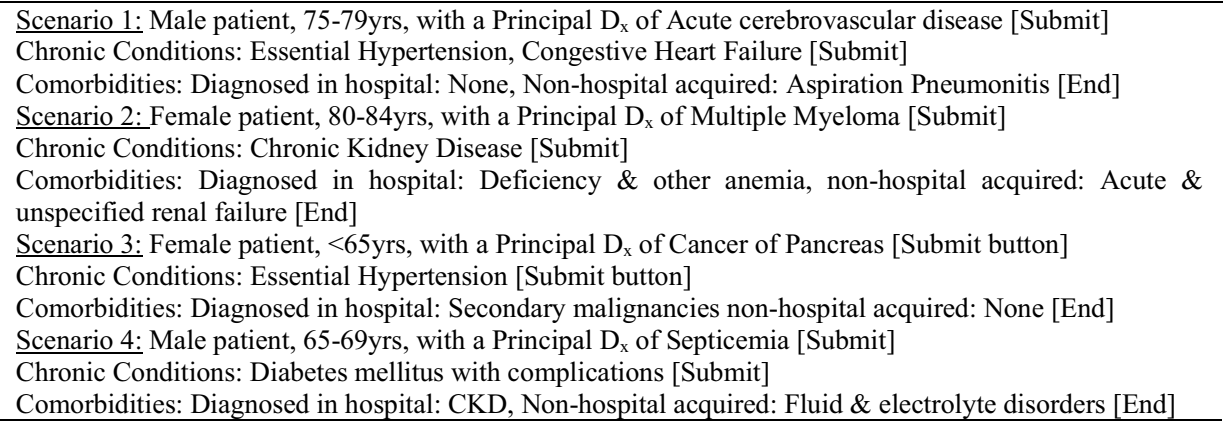




\section{Results}

\subsection{Univariate Analysis}

The participants had an equal gender distribution ( 9 male, 9 female). Their mean age was 28.2 years (std. dev. $=1.7$ years). Half of the participants answered that they are very familiar with medical simulation systems. When asked about their perceived expertise with computer use, seven participants (38.9\%) said that they had average expertise, nine (50\%) above average, and two participants $(11.1 \%)$ thought that they have a far above average expertise. Finally, seven participants (38.9\%) were somewhat familiar, and only two participants $(11.1 \%)$ not as familiar with medical simulation systems. Of the four evaluation dimensions, the one with the highest score was the 'system features' (4.15); the dimension with the lower score was the 'information validity' (3.77). The composite variable about the participants' opinion on data-driven education was found to be 4.27.

Table 2. Individual item responses, Internal Validity and Composite Scores

\begin{tabular}{|c|c|c|c|}
\hline & $\begin{array}{c}\text { Mean } \\
\text { (s.d) }\end{array}$ & $\begin{array}{l}\text { Cronbac } \\
\text { h Alpha }\end{array}$ & $\begin{array}{l}\text { Composite } \\
\text { Mean (s.d) }\end{array}$ \\
\hline \multicolumn{4}{|l|}{ Dimension 1: System Navigation } \\
\hline The software that I used today was easy to navigate & $3.89(.83)$ & \multirow{5}{*}{.70} & \multirow{5}{*}{$3.96(.47)$} \\
\hline I could navigate the software without any problems & $3.89(.96)$ & & \\
\hline I could understand the available choices that I was provided & $4.17(.51)$ & & \\
\hline The navigation buttons were placed where they should be & $4.17(.51)$ & & \\
\hline It was easy to select options from drop-down lists & $3.72(.57)$ & & \\
\hline \multicolumn{4}{|l|}{ Dimension 2: System Usefulness } \\
\hline Provided relevant information about comorbidities & $3.94(.63)$ & \multirow{5}{*}{.87} & \multirow{5}{*}{$3.98(.47)$} \\
\hline Provided useful insights of the comorbidity effect on outcomes & $3.89(.83)$ & & \\
\hline The information of the system was interesting & $4.11(.47)$ & & \\
\hline The system can help learn about the impact of comorbidities & $4.06(.53)$ & & \\
\hline Provides feedback \& information relevant in actual clinical cases & $3.94(.41)$ & & \\
\hline \multicolumn{4}{|l|}{ Dimension 3: Validity of information } \\
\hline The information that the system provided was correct & $3.83(.51)$ & \multirow{3}{*}{.82} & \multirow{3}{*}{$3.77(.54)$} \\
\hline The information that the system provided was accurate & $3.78(.64)$ & & \\
\hline The information that the system provided was unambiguous & $3.72(.75)$ & & \\
\hline \multicolumn{4}{|l|}{ Dimension 4: System Features } \\
\hline Separating chronic vs hospital acquired conditions was important & $4.17(.51)$ & \multirow{4}{*}{.90} & \multirow{4}{*}{$4.15(.46)$} \\
\hline $\begin{array}{l}\text { The step-by-step system approach to develop my scenario was } \\
\text { helpful }\end{array}$ & $4.17(.51)$ & & \\
\hline The summary of the scenarios after completion is a useful feature & $4.00(.48)$ & & \\
\hline The updated risk estimations output after each step is a useful feature & $4.28(.57)$ & & \\
\hline \multicolumn{4}{|l|}{ Opinion about data-driven medical education } \\
\hline Data-driven simulations systems are needed in medical curricula & $4.22(.54)$ & \multirow{4}{*}{.84} & \multirow{4}{*}{$4.27(.40)$} \\
\hline Big data provide opportunities to examine clinical case combinations & $4.39(.50)$ & & \\
\hline Comorb. with varying effect on outcomes should be easy to navigate & $4.28(.46)$ & & \\
\hline Familiarizing with data-driven estimations help proactive decisions & $4.22(.42)$ & & \\
\hline
\end{tabular}

\subsection{External Factors Associated with Perceptions about TrainComorb}

Bivariate analysis was conducted to examine the association between the participants' age, their self-reported computer experience, and the simulation system familiarity, with each of the four composite evaluation dimensions. A negative and statistically significant correlation was observed between the participants' age and (i) their responses on the 'information validity' dimension $(\mathrm{r}=-0.44, \mathrm{p}<0.10)$ and (ii) their opinion regarding data driven education $(\mathrm{r}=-0.52, \mathrm{p}<0.05)$. The older the residents were, the more skeptical they 
appeared to be regarding computer-generated clinical information, and they had a less positive opinion about the potential in data driven education.

A positive statistical association was observed between the familiarity with simulation systems and the 'information validity' dimension $(r=0.49, \mathrm{p}<0.05)$ : Those exposed more in medical simulation systems appeared to be more confident about the information that the system was generating. Similarly, a positive association was observed between the residents' opinion about data-driven education and the 'system usefulness' $(\mathrm{r}=0.44, \mathrm{p}<0.1)$ and the 'system features' $(\mathrm{r}=0.62, \mathrm{p}<0.01)$ dimensions. No correlation was observed between the reported computer experience and any of the examined dimensions (Table 3).

Table 3. Association of External Factors with Perceptions about TrainComorb

\begin{tabular}{lccccc}
\hline & $\begin{array}{c}\text { System } \\
\text { Navigation }\end{array}$ & $\begin{array}{c}\text { System } \\
\text { Usefulness }\end{array}$ & $\begin{array}{c}\text { Information } \\
\text { Validity }\end{array}$ & $\begin{array}{c}\text { System } \\
\text { Features }\end{array}$ & $\begin{array}{c}\text { Opinion Data- } \\
\text { Driven educ. }\end{array}$ \\
\hline Age & $0.03(\mathrm{p}=.99)$ & $-0.34(\mathrm{p}=.17)$ & $-0.44(\mathrm{p}=.07)$ & $-0.36(\mathrm{p}=.15)$ & $-0.52(\mathrm{p}=.03)$ \\
Computer Experience & $-0.21(\mathrm{p}=.39)$ & $-0.19(\mathrm{p}=.44)$ & $0.14(\mathrm{p}=.57)$ & $-0.09(\mathrm{p}=.71)$ & $-0.02(\mathrm{p}=.92)$ \\
Familiar with sim systems & $-0.10(\mathrm{p}=.69)$ & $-0.05(\mathrm{p}=.82)$ & $0.49(\mathrm{p}=.03)$ & $-0.01(\mathrm{p}=.96)$ & $-0.09(\mathrm{p}=.71)$ \\
Opinion Data-Driven educ. & $0.34(\mathrm{p}=.16)$ & $0.44(\mathrm{p}=.06)$ & $0.16(\mathrm{p}=.51)$ & $0.62(\mathrm{p}=.01)$ & \\
\hline
\end{tabular}

\section{Conclusions}

The medical residents evaluated the four dimensions with average scores between 3.77 and 4.15 (5-scale) and thought highly of data-driven medical education. The finding supports a recently acknowledged need for data-driven physicians [6]. Although the residents' evaluation was not associated with their computer experience, those more familiar with simulation systems, and more positive about data-driven education, evaluated the dimensions 'usefulness', 'validity', and 'features' with higher scores: Apparently, the role of experience in user perception has been discussed in the literature, as in [7]. The new generation of medical students are gradually becoming familiar with alternative modes of medical training, and they appear to value evidence based and data driven modalities as useful supplementary learning approaches. This is supported by the external factor associations that were observed in the present study.

TrainComorb has the potential to become a useful supplementary tool for the education of future medical professionals. The results of this user-based evaluation study and the system prototype itself, are being communicated with the upper administration of a College of Medicine and its Simulation Center in the region of central Michigan, to identify effective venues of integration with the formal medical curricula.

\section{References}

[1] Van Weel C, Schellevis FG, Comorbidity and guidelines: Conflicting interests, Lancet 18 (2006), 550-1.

[2] Zikos D, Ragina N, Strong O, Stud Health Technol Inform 272 (2020), 83-86.

[3] Nykänena P, Brender J, Talmonc J, et al., Guideline for good evaluation practice in health informatics (GEP-HI). Int. J. Med. Inf. 80 (2011), 815-27.

[4] National Career Development Association, Software evaluation criteria, https://tinyurl.com/2725a9je

[5] IBM Corp, IBM SPSS Statistics for Windows (2019), Version 26.0. Armonk, NY: IBM Corp.

[6] Stanford Medicine 2020 Health Trends Report, The Rise of the Data-Driven Physician, (2020), Available at: https://med.stanford.edu/dean/healthtrends.html

[7] Ribeiro R, The Role of Experience in Perception. Human Studies 37 (2014), 559-81. 